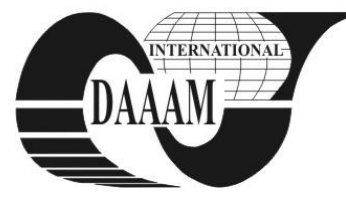

Annals of DAAAM for 2011 \& Proceedings of the 22nd International DAAAM Symposium, Volume 22, No. 1, ISSN 1726-9679 ISBN 978-3-901509-83-4, Editor B. Katalinic, Published by DAAAM International, Vienna, Austria, EU, 2011 Make Harmony between Technology and Nature, and Your Mind will Fly Free as a Bird

\title{
MANUFACTURING SYSTEMS MODELLING AND SIMULATION SOFTWARE DESIGN: A REFERENCE MODEL
}

\author{
BATTISTA, C[laudia]; DELLO STRITTO, G[iulia]; GIORDANO, F[rancesco]; IANNONE, R[affaele] \& \\ SCHIRALDI, M[assimiliano] M.
}

\begin{abstract}
Nowadays production environments request standard efficient frameworks in order to map and simulate manufacturing processes which literature or market can barely provide. A generalized reference model is necessary to highlight the differences and affinities among manufacturing systems resources, so as to examine the possibilities of modelling of such concepts and their respective roles. OPUS project formalizes a new modelling and simulation language for productive manufacturing systems through the support of an optimization tool development. On a practical level a BPMN approach has been useful to conceive a model strictly related to business processes, in addition to the simplification of the implementation/coding of the simulation tool.

Key words: manufacturing systems, reference model, simulation, software architecture
\end{abstract}

\section{INTRODUCTION}

Simulation is considered a useful tool in order to study and optimize production processes. Several authors agree on simulation potentialities in the analysis of the dynamic and stochastic behaviour of manufacturing system; they also predict its operational performance and point out its critical factors (Smith, 2003; Law, 2007). However, literature informs about the absence of a commercial software which incorporates all the strategic functionalities for the modelling of several manufacturing phases, in a user-friendly way.. Although simulation is always perceived as one of the best approaches in order to improve system efficiency (Mohora, 2009), there are some limits which seem to prevent the diffusion of such tools in manufacturing enterprises (Rogers, 2002).

If on the one hand the conceptual modelling is one of the most vital parts in a simulation study, on the other hand it is one of the least understood. A strong need for a steady reference model is outlined in the manufacturing background (Nylund, 2008). At present, most of the simulation software available on the market implements a graphical model-building approach (Van Beek et al., 2008) - the so called "development environment" - where experienced users are able to model almost any type of process through basic function blocks; some user-defined statistical functions, then, evaluate the whole system behaviour. Occasionally, formal meta-languages are used to describe the relationships among the components (resources, entities, etc.). Moreover, advanced modelling simulation knowledge and skills are always required.

On the contrary, a user-friendly simulation software becomes critical with the absence of a strong competence in operations research or statistics, which constitute the traditional background of the analysis in industrial companies. Several authors (Bodner and Mc Ginnis, 2002; Narayanan et al., 1998) identify the necessity of a standard reference framework for the development of production models and logistics processes as a success factor for the wide diffusion of simulation softwares in manufacturing industry. Some important issues must be considered, such as the definition of conceptual model and conceptual model requirements, conceptual model development methodology, conceptual model representation and communication rules (Robinson, 2006).

A modelling language needs to be designed so as to facilitate the simulation software development. In this regard, the Università degli Studi di Tor Vergata and Università degli Studi di Salerno realized a reference model to supply a structural approach for the schematization of the manufacturing environment in order to improve performance operations. This reference model (developed within the OPUS project) may consitute a standard to represent resources and management policies in manufacturing systems; moreover, it is specifically suitable to simplify the implementation of the simulation model as well as to support the simulation software development.

\section{THE REFERENCE MODEL}

The elements which constitute the reference model are shown in fig. 1 (model hierarchy). In particular, the structure includes the following layers:

1. Logic layer

2. Communication layer

3. Operations layer

The "logic layer" contains information about the bill of material (BoM), the process chart (PC), the master production schedule (MPS), the management policies, etc., and allows to gather a structured description of the logic behind material flows and resources allocation. Tab. 1 (PC) gives an example of the information contained in the PC record.

The "communication layer" integrated in the model allows the coordination of resources according to the specification of the logic layer. It has been developed using a BPMN representation in order to standardize processes and to simplify their construction. Such processes facilitate interactions among resources belonging to the subsequent operations layer.

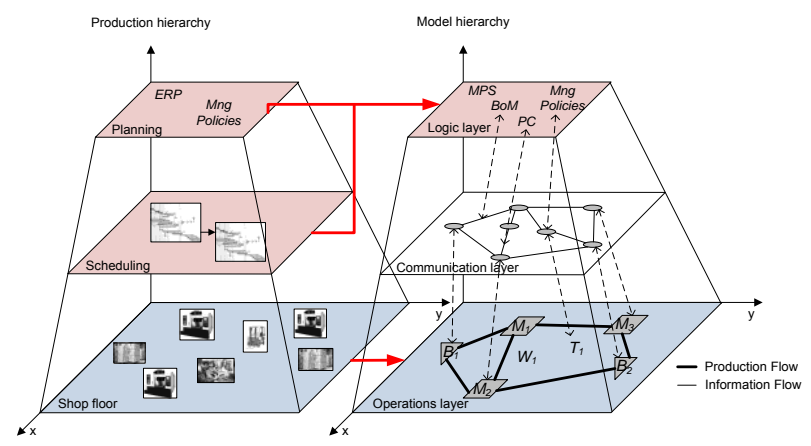

Fig. 1. Reference model hierarchy

The "operations layer" allows to reproduce the resources behaviour, the production layout and the material flows. The layout is shaped through 4 different kinds of meta-models: machines (M), buffers (B), transporters (T), workers (W). It is necessary to define such resources by position and technical features. Tab. $1\left(\mathrm{M}_{\mathrm{i}}\right)$ shows an example of the parameters and 
the functions used in the definition and the performance of the machines.

\begin{tabular}{|l|}
\hline PC parameters \\
\hline pk part number \\
\hline pk phase \\
\hline Description \\
\hline pk machine \\
\hline pk worker \\
\hline Scraps \\
\hline run time \\
\hline lot dimension \\
\hline
\end{tabular}

\begin{tabular}{|l|}
\hline $\mathbf{M}_{\mathbf{i}}$ parameters \\
\hline pk name \\
\hline Type \\
\hline std production rate \\
\hline Capacity \\
\hline layout position (x,y) \\
\hline Size \\
\hline pk failure \\
\hline pk std calendar \\
\hline pk buffer in \\
\hline pk buffer out \\
\hline depreciation charge \\
\hline nominal power \\
\hline pk setup time matrix \\
\hline
\end{tabular}

\begin{tabular}{|l|}
\hline $\mathbf{M}_{\mathbf{i}}$ functions \\
\hline material_request() \\
\hline material_load() \\
\hline resource_request() \\
\hline produce() \\
\hline release() \\
\hline setup() \\
\hline
\end{tabular}

Tab. 1. Process chart (PC) and Machine $\left(\mathrm{M}_{\mathrm{i}}\right)$ meta-models

The usage and the readjustment of Habchi \& Berchet (2003) model in order to describe the reference model (fig. 1), clarify that the construction of the hierarchical structure is aimed at the standardization and simplification of the production environment schema, and at a facilitation of the model implementation. The logic layer contains the information to reproduce management policies, while the operation layer includes the data about resources. The aim of such a distinction is to completely separate the model functioning logic (which derives data from the management information system) from the physical part (constructed with shop floor data).

\subsection{Innovative aspects}

The model suggested allows simulating manufacturing processes in accordance to the main operations management theories. Its innovative aspects are based on five main pillars:

- the inputs originate exclusively from the typical manufacturing systems data structures: Bills Of Materials, Master Production Schedules, Process Charts, etc.;

- the basic production processes archetypes (i.e. set-ups, machine failures, etc.) are natively supported;

- $\quad$ the main production and inventory management policies (i.e. Re-Order Level policies, Material Requirement Planning, etc.) are natively supported;

- $\quad$ there is a clear distinction between the physical and the information layer; the user needs to designe only the former, since the data structure will provide all the required information to complete the model flows (e.g. the items processing sequence information are already defined into the process charts);

- $\quad$ some data structures (lists) coordinate any informative and physical flux which pertains to the model resources.

Thus, the user is able to build models simply through the filling of the data structures (which are formalized in the reference model). It is not required to build any product paths linking the resources or creating fictitious entity generation nodes, differently from other simulation tools.

\section{SOFTWARE ARCHITECTURE}

The development of this reference model has already led to design a software simulation tool (O.P.U.S. - Optimizing Production Using Simulation) which focuses on flexibility and simplicity of use.

BPMN approach allows to represent the company business processes - to an extremely detailed level - and supports the manufacturing framework embedded in OPUS. It has been implemented in Java language according to the following rules: the shop floor functions (i.e. a machinery may send a material picking request to a stock buffer or may send a confirmation message for a material release to downstream resources) have been directly translated into the Java classes and methods of the related objects, in order to obtain a complete compliance among the simulation logic and the real shop floor environments.

Other peculiarities are: simplicity of use due to a modelling wizard; functions to identify cause-effect relations and critical process phases; optimization analysis suggesting the most efficient solutions.

Currently the software kernel (in "command line" version) of the OPUS simulator is available for testing. An open source version and a full GUI version (distributed free of charge to Italian Universities) will be available in a few months, in order to support operations management teaching.

\section{CONCLUSION}

OPUS project supplies a reference framework in order to represent a standard to model resources interactions and their relationships in manufacturing systems. A simulation tool must provide a standard modelling and simulation language.

Nowadays many enterprises usually design their own business processes with an intensive usage of BPMN, thus to support all the company production and logistics functions representation through an innovative approach. An enterprise which requests a business process assessment can find an opportunity to develop economical and technical indicators as well as a Decision Support System. Further researches will be focused on the introduction of this modelling and simulation tool directly in the manufacturing real environment, in order to evaluate the effectiveness of the model.

\section{REFERENCES}

Bodner, D. A. \& L. F. McGinnis (2002). A structured approach to simulation modeling of manufacturing systems, Proceedings of the 2002 Industrial Engineering Research Conference, Georgia

Habchi, G.; Berchet, C. (2002). A model for manufacturing systems simulation with a control dimension. Simulation Modelling Practice and Theory, Vol. 11 (2003) 21-44, 1569-190X

Law, A. a. (2007). Simulation modelling and analysis, $4^{\text {th }}$ edition, McGraw-Hill, ISBN: 9780072988437, New York

Mohora, C.; Anania, D. \& Calin, O. A. (2009). Simulations Strategies using Delmia Quest, Annals of DAAAM 2009, 25-28th November 2009, pp.335-336, ISSN 1726-9679, ISBN 978-3-901509-70-4, Vienna, Austria

Narayanan, S.; Bodner, D.A.; Sreekanth, U.; Govindaraj, T.; McGinnis, L.F. \& Mitchell, C.M. (1998). Research in object-oriented manufacturing simulations: an assessment of the state of the art, IIE Transactions, Vol.30, No.9, ISSN: 795-810

Nylund, H.; Salminen, K. \& Andersson, P. (2008). Modelling and Simulation in the Context of Distributed Manufacturing Systems, Annals of DAAAM 2008, pp.927-928, 22-25th October 2008, ISSN 1726-9679, Trnava, Slovakia

Robinson S. (2006). Conceptual modeling for simulation: issues and research requirements, Proceedings of the 2006 Winter Simulation Conference, IEEE, ISBN: 1-4244-05009, Piscataway, NJ

Rogers, P. (2002). Simulation of manufacturing operations: optimum-seeking simulation in the design and control of manufacturing systems experience with optquest for arena, Proceedings of the 34th conference on Winter simulation: exploring new frontiers, ISBN:0-7803-7615-3, pp. 11421150, San Diego, California, United States

Smith, J. (2003). Survey of the use of simulation for manufacturing system design and operation, Journal of manufacturing systems, Vol.22, No.2, pp.157-171

Van Beek D.A.; Hofkamp, A.T.; Reniers, M.A.; Rooda J.E. \& Schiffelers R.R.H. (2008). Syntax and Formal Semantics of Chi 2.0, ISSN: 1872-1567 